\title{
Synthesis of 2,6-Diaryl-4-Indolylpyridines as Novel 5-LOX Inhibitors
}

Berihu Tekluu ${ }^{1}$, Sunanda Kumari Kadiri ${ }^{2}$ and Siddaiah Vidavalur ${ }^{1 *}$

${ }^{1}$ Department of Organic Chemistry and FDW, College of Science and Technology, Andhra University, Visakhapatnam, Andhra Pradesh, India

${ }^{2}$ Department of Microbiology, College of Science and Technology, Andhra University, Visakhapatnam, Andhra Pradesh, India

\begin{abstract}
A series of 2,6-diaryl substituted -4-indolylpyridines have been synthesized from indole-3-carboxaldehyde and acetophenones and all the compounds characterized by spectroscopic techniques. 5-Lipoxygenase enzyme inhibitory activities were performed for all the compounds. Among the 2, 6-diaryl substituted -4-indolylpyridine derivatives 3ad and 3aa showed good activity.
\end{abstract}

Keywords: Indolylpyridine; 5-LOX; Indole-3-carboxaldehyde

\section{Introduction}

3-Substituted indole is a privileged structural motif found in many biologically active compounds and natural products [1]. 3-Substituted indole derivatives exhibit several biological activities such as antibacterial [2-6], anti-inflammatory [7-10], antitumor [1113], anticancer [14-18], anti-hypertensive [19], anti-depressant [20,21] and antiviral [22-25] activities. On the other hand, the molecules having pyridine nucleus possess a large spectrum of biological activities like anti-prion [26], anti-hepatitis B virus [27], antibacterial [28], anticancer [29] and antimalarial [30] activities. Therefore, the combined molecules of 3-Substituted indole and pyridine frame works, indolylpyridines, are the valuable starting material for the synthesis of structurally diverse biologically active agents. Indolylpyridines have been reported to exhibit several biological activities such as anti-cancer and anti-inflammatory activities [31,32]. However, 5-lipoxygenase enzyme inhibitory activity (5-LOX) of indolylpyridines has not been fully explored. 5-Lipoxygenase is the key enzyme for the biosynthesis of leukotrienes, the important mediators for inflammatory, allergic, and obstructive processes. 5-LOX inhibitors have potential in treating asthma and various inflammatory disorders $[33,34]$. Therefore, herein we report the synthesis of a series of 2,6-diaryl-4-indolylpyridines from substituted acetophenones and $1 \mathrm{H}$-indole-3-carbaldehydes using ammonium acetate as a nitrogen source in the presence of acetic acid and 5-LOX activities of several 2, 6-diaryl-4-indolylpyridines.

\section{Experimental Section}

\section{General}

All the chemicals used were of synthetic grade procured from Sigma Aldrich. Completion of the reactions was monitored by analytical thin layer chromatography (TLC) using E-Merck $0.25 \mathrm{~mm}$ silica gel plates using ethyl acetate/hexane as solvent system, visualization was accomplished with UV light $(256 \mathrm{~nm})$ and iodine chamber. Synthesized compounds were purified by column chromatography (silica gel 100200 mesh) using a mixture of hexane and ethyl acetate. Melting points were measured in open capillary tubes and were uncorrected; all the ${ }^{1} \mathrm{H}$ and ${ }^{13} \mathrm{C}$ spectra were recorded in $\mathrm{CDCl}_{3}$ solvent $\left(400 \mathrm{MHz}\right.$ for ${ }^{1} \mathrm{H}$ and $100 \mathrm{MHz}$ for ${ }^{13} \mathrm{C}$ ) relative to TMS internal standard, proton coupling patterns were described as singlet (s), doublet (d), triplet ( $\mathrm{t}$ ), quartet (q) and multiplet $(\mathrm{m})$. The electron ionization mass spectra were recorded on Agilent 1100.

General experimental procedure for the synthesis of 1H-indole-3-carboxaldehydes (1a or $1 \mathrm{~b}$ )

To a solution of substituted indole $(42.6 \mathrm{mmol})$ (or 5-bromo indole) in dry DMF (187.4 mmol) in an ice-salt bath, $\mathrm{POCl}_{3}(47.1 \mathrm{mmol})$ was subsequently added with stirring over a period of $30 \mathrm{~min}$. After completion of addition, the temperature was raised to $40^{\circ} \mathrm{C}$, the syrup was stirred for $1.5 \mathrm{~h}$ at same temperature. At the end of the reaction (as indicated by TLC) $25 \mathrm{gms}$ crushed ice was added to the reaction mixture. The obtained solution was transferred into $250 \mathrm{~mL}$ RB flask, $\mathrm{NaOH}$ ( $470 \mathrm{mmol}$ ) dissolved in $50 \mathrm{~mL}$ water was added with constant stirring and the resultant suspension was heated rapidly to the boiling point and allowed to cool to room temperature. The mixture was allowed to stand in refrigerator overnight. The precipitate was filtered off, washed thrice with $100 \mathrm{~mL}$ water, yielding $1 \mathrm{H}$-indole-3-carboxaldehydes (1a or $1 \mathrm{~b}$ ).

1H-Indole-3-carboxaldehyde (1a): Brownish yellow solid, Yield: 92\%, Mp: $196-198^{\circ} \mathrm{C},{ }^{1} \mathrm{H}$ NMR (DMSO- $d, 400 \mathrm{MHz}$ ): $\delta=9.52(\mathrm{~s}, 1 \mathrm{H})$, $8.12(\mathrm{~s}, 1 \mathrm{H}), 7.62(\mathrm{~d}, 1 \mathrm{H}), 7.52(\mathrm{~s}, 1 \mathrm{H}), 7.34(\mathrm{~d}, 1 \mathrm{H}), 7.22(\mathrm{t}, 1 \mathrm{H}), 7.14(\mathrm{t}$ $1 \mathrm{H}) .{ }^{13} \mathrm{C}$ NMR (DMSO- $\left.d_{6}, 100 \mathrm{MHz}\right): \delta=1882.7,137.2,131.82,127.7$, $122.4,120.5,119.4,118.0,111.4$.

Bromo-1H-indole-3-carboxaldehyde (1b): Cream coloured solid, Yield: $90 \%$, Mp: $192^{\circ} \mathrm{C},{ }^{1} \mathrm{H}$ NMR (DMSO- $d_{6}, 400 \mathrm{MHz}$ ): $\delta=9.94(\mathrm{~s}, 1 \mathrm{H})$, $8.32(\mathrm{~s}, 1 \mathrm{H}), 8.25(\mathrm{~s}, 1 \mathrm{H}), 7.75(\mathrm{~s}, 1 \mathrm{H}), 7.43(\mathrm{~d}, 1 \mathrm{H}), 7.34(\mathrm{~d}, 1 \mathrm{H}) \cdot{ }^{13} \mathrm{C}$ NMR (DMSO- $\left.d_{6}, 100 \mathrm{MHz}\right): \delta=183.9,144.4,136.7,135.2,125.6,123.1$, $117.3,114.8,113.0$

\section{General experimental procedure for synthesis of 2,6-diaryl- 4-indolylpyridines}

A mixture of $1 \mathrm{H}$-indole-3-carboxaldehyde (1) $(1.0 \mathrm{mmol})$ and acetophenone (2) $(2.0 \mathrm{mmol})$ in the presence of $\mathrm{AcONH}_{4}(5 \mathrm{~mol} \%)$ and acetic acid was heated in an oil bath at reflux for about $5 \mathrm{~h}$. After the completion of the reaction (as monitored by TLC), the reaction mixture was cooled to room temperature and partitioned between water and ethyl acetate. The organic layer was separated and dried over anhydrous sodium sulphate and concentrated under vacuum to afford the crude compound. The crude compound was purified with silica gel column chromatography using hexane/EtOAc as eluents to afford the pure product (3) (Supplementary Figures 1-18).

${ }^{*}$ Corresponding author: Siddaiah Vidavalur, Department of Organic Chemistry and FDW, College of Science and Technology, Andhra University, Visakhapatnam, Andhra Pradesh, India, Tel: 089128 44000; E-mail: sidduchem@gmail.com

Received May 24, 2017; Accepted May 27, 2017; Published May 31, 2017

Citation: Tekluu B, Kadiri SK, Vidavalur S (2017) Synthesis of 2,6-Diaryl-4Indolylpyridines as Novel 5-LOX Inhibitors. Med Chem (Los Angeles) 7: 894-899. doi: 10.4172/2161-0444.1000449

Copyright: ( 2017 Tekluu B, et al. This is an open-access article distributed under the terms of the Creative Commons Attribution License, which permits unrestricted use, distribution, and reproduction in any medium, provided the original author and source are credited. 
Citation: Tekluu B, Kadiri SK, Vidavalur S (2017) Synthesis of 2,6-Diaryl-4-Indolylpyridines as Novel 5-LOX Inhibitors. Med Chem (Los Angeles) 7: 894-899. doi: 10.4172/2161-0444.1000449

\section{Characterization of 2,6-diaryl-4-indolylpyridines}

3-(2,6-di (Phenylpyridin-4-yl)-1H-indole (3aa): Colorless solid, Yield: $80 \%$, Mp: $178-180^{\circ} \mathrm{C},{ }^{1} \mathrm{H}$ NMR $\left(400 \mathrm{MHz}, \mathrm{CDCl}_{3}\right): \delta=8.71(\mathrm{~d}$, $1 \mathrm{H}), 8.23(\mathrm{~d}, 4 \mathrm{H}), 8.08(\mathrm{~d}, 1 \mathrm{H}), 7.99(\mathrm{~s}, 2 \mathrm{H}), 7.60(\mathrm{~d}, 1 \mathrm{H}), 7.55(\mathrm{~m}, 4 \mathrm{H})$, 7.48 (d, 3H), 7.31 (t, 2H). ${ }^{13} \mathrm{C}$ NMR $\left(100 \mathrm{MHz}, \mathrm{CDCl}_{3}\right): \delta=157.3,145.1$, $139.6,136.9,129.0,128.7,127.2,125.3,123.6,122.9,121.1,119.6,117.1$ 116.0, 111.9. HRMS (ESI): $\mathrm{m} / \mathrm{z}[\mathrm{M}+\mathrm{H}]^{+}$calcd for $\mathrm{C}_{25} \mathrm{H}_{18} \mathrm{~N}_{2}$ : found: 347.2.

3-[2,6-di(p-Tolyl)pyridin-4-yl]-1H-indole (3ab): Colorless solid, Yield: $75 \%$, Mp: $218-220^{\circ} \mathrm{C},{ }^{1} \mathrm{H}$ NMR $\left(400 \mathrm{MHz}, \mathrm{CDCl}_{3}\right): \delta=8.63(\mathrm{~d}$, $1 \mathrm{H}), 8.24(\mathrm{~d}, 4 \mathrm{H}), 7.91(\mathrm{~d}, 1 \mathrm{H}), 7.82(\mathrm{~d}, 1 \mathrm{H}), 7.35(\mathrm{~s}, 2 \mathrm{H}), 7.28(\mathrm{~d}, 4 \mathrm{H})$, $7.22(\mathrm{~d}, 1 \mathrm{H}), 7.16(\mathrm{t}, 2 \mathrm{H}), 2.3(\mathrm{~d}, 6 \mathrm{H}) .{ }^{13} \mathrm{C}$ NMR $\left(100 \mathrm{MHz}, \mathrm{CDCl}_{3}\right)$ : $\delta=155.6,144.5,138.3,135.5,135.3,129.0,128.1,126.6,126.0,124.5$, $120.0,116.9,115.5,112.3,111.0,21.3$. HRMS (ESI): $\mathrm{m} / \mathrm{z}[\mathrm{M}+\mathrm{H}]^{+} \mathrm{calcd}$ for $\mathrm{C}_{27} \mathrm{H}_{22} \mathrm{~N}_{2}$ : found: 375.8 .

3-[2,6-bis(4-Methoxyphenyl)pyridin-4-yl]-1H-indole (3ac): Colorless solid, Yield: $80 \%$, Mp: $230-232^{\circ} \mathrm{C},{ }^{1} \mathrm{H}$ NMR $(400 \mathrm{MHz}$, $\mathrm{CDCl}_{3}$ ): $\delta=8.65(\mathrm{~d}, 1 \mathrm{H}), 8.25(\mathrm{~d}, 4 \mathrm{H}), 7.94(\mathrm{~d}, 1 \mathrm{H}), 7.86(\mathrm{~d}, 1 \mathrm{H}), 7.39(\mathrm{~s}$, $2 \mathrm{H}), 7.29(\mathrm{~d}, 4 \mathrm{H}), 7.25(\mathrm{~d}, 1 \mathrm{H}), 7.18(\mathrm{t}, 2 \mathrm{H}), 3.8(\mathrm{~s}, 6 \mathrm{H}) .{ }^{13} \mathrm{C}$ NMR $(100$ $\mathrm{MHz}, \mathrm{CDCl}_{3}$ ): $\delta=156.2,144.1,137.6,135.2,134.9,130.6,128.5,126.8$, 125.1, 122.8, 119.6, 115.5, 114.9, 112.7, 111.2, 55.8. HRMS (ESI): $\mathrm{m} / \mathrm{z}$ $[\mathrm{M}+\mathrm{H}]^{+}$calcd for $\mathrm{C}_{27} \mathrm{H}_{22} \mathrm{~N}_{2} \mathrm{O}_{2}$ : found: 407.8.

3-[2,6-bis(4-Chlorophenyl)pyridin-4-yl]-1H-indole (3ad): White solid, Yield: $84 \%$, Mp: $186-188^{\circ} \mathrm{C},{ }^{1} \mathrm{H}$ NMR $\left(400 \mathrm{MHz}, \mathrm{CDCl}_{3}\right)$ : $\delta=8.61(\mathrm{~d}, 1 \mathrm{H}), 8.21(\mathrm{~d}, 4 \mathrm{H}), 7.93(\mathrm{~d}, 1 \mathrm{H}), 7.88(\mathrm{~d}, 1 \mathrm{H}), 7.40(\mathrm{~s}, 2 \mathrm{H})$, $7.32(\mathrm{~d}, 4 \mathrm{H}), 7.29$ (d, $1 \mathrm{H}), 7.21(\mathrm{t}, 2 \mathrm{H}) .{ }^{13} \mathrm{C}$ NMR $\left(100 \mathrm{MHz}, \mathrm{CDCl}_{3}\right)$ : $\delta=155.8,144.5,138.2,135.4,135.2,128.9,128.3,126.9,126.0,124.2$, $120.1,116.9,115.8,112.5,111.2$. HRMS (ESI): $\mathrm{m} / \mathrm{z}[\mathrm{M}+\mathrm{H}]^{+} \mathrm{calcd}$ for $\mathrm{C}_{25} \mathrm{H}_{16} \mathrm{~N}_{2} \mathrm{Cl}_{2}$ : found: 416.7 .

3-[2,6-bis(4-Bromophenyl)pyridin-4-yl]-1H-indole

(3ae): Colorless solid, Yield: $82 \%$, Mp: $216-217^{\circ} \mathrm{C},{ }^{1} \mathrm{H}$ NMR $(400 \mathrm{MHz}$, $\left.\mathrm{CDCl}_{3}\right): \delta=8.58(\mathrm{~d}, 1 \mathrm{H}), 8.07(\mathrm{~d}, 4 \mathrm{H}) ; 7.86(\mathrm{~d}, 1 \mathrm{H}) 7.59(\mathrm{~d}, 4 \mathrm{H}), 7.52$ (s, 2H), $7.45(\mathrm{~d}, 1 \mathrm{H}) 7.39$ (d, $1 \mathrm{H}), 7.21(\mathrm{t}, 2 \mathrm{H}) .{ }^{13} \mathrm{C}$ NMR $(100 \mathrm{MHz}$, $\left.\mathrm{CDCl}_{3}\right): \delta=156.6,144.7,138.6,134.6,130.8,129.1,125.8,124.2,122.8$, $119.7,119.1,117.3,118.5,115.5,113.4$. HRMS (ESI): $\mathrm{m} / \mathrm{z}[\mathrm{M}+\mathrm{H}]^{+} \mathrm{calcd}$ for $\mathrm{C}_{25} \mathrm{H}_{16} \mathrm{~N}_{2} \mathrm{Br}_{2}$ : found: 505.7 .

3-(2,6-bis(4-Fluorophenyl)pyridin-4-yl)-1H-indole (3af): White Solid, Yield: $75 \%$, Mp: $175-177^{\circ} \mathrm{C},{ }^{1} \mathrm{H}$ NMR $\left(400 \mathrm{MHz}, \mathrm{CDCl}_{3}\right): \delta=8.51$ (d, 1H), 8.22 (d, 4H), 8.19 (d, 1H), 7.95 (s, 2H), 7.65 (d, 1H), $7.52(\mathrm{~d}$, $1 \mathrm{H}), 7.33$ (t, 2H), 7.29 (d, $4 \mathrm{H}) .{ }^{13} \mathrm{C}$ NMR $\left(100 \mathrm{MHz}, \mathrm{CDCl}_{3}\right): \delta=164.8$, $156.4,145.0,136.8,135.9,128.9,125.2,123.3,121.2,119.6,116.6,116.1$, 115.7, 115.4, 111.8. HRMS (ESI): $\mathrm{m} / \mathrm{z}[\mathrm{M}+\mathrm{H}]^{+}$calcd for $\mathrm{C}_{25} \mathrm{H}_{16} \mathrm{~N}_{2} \mathrm{~F}_{2}$ : found: 383.

3-[2,6-di(Pyridin-4-yl)pyridin-4-yl]-1H-indole (3ag): Colorless solid, Yield: $63 \%$, Mp: $378-380^{\circ} \mathrm{C},{ }^{1} \mathrm{H}$ NMR $\left(400 \mathrm{MHz}, \mathrm{CDCl}_{3}\right): \delta=9.24$ $(\mathrm{d}, 1 \mathrm{H}), 8.77(\mathrm{~d}, 4 \mathrm{H}), 8.42(\mathrm{~d}, 4 \mathrm{H}), 8.40(\mathrm{~s}, 2 \mathrm{H}), 7.93(\mathrm{~s}, 1 \mathrm{H}), 7.61(\mathrm{~d}$, $1 \mathrm{H}), 7.50(\mathrm{~m}, 1 \mathrm{H}), 7.22(\mathrm{~m}, 2 \mathrm{H}) .{ }^{13} \mathrm{C} \mathrm{NMR}\left(100 \mathrm{MHz}, \mathrm{CDCl}_{3}\right): \delta=155.5$, 151.1, 146.4, 145.8, 137.2, 129.7, 127.0, 122.3, 121.1, 120.4, 119.1, 118.1, 113.0, 102.8. HRMS (ESI): $\mathrm{m} / \mathrm{z}[\mathrm{M}+\mathrm{H}]^{+}$calcd for $\mathrm{C}_{23} \mathrm{H}_{16} \mathrm{~N}_{4}$ : found: 349.8 .

3-[2,6-di(Furan-2-yl)pyridin-4-yl]-1H-indole(3ah): White solid, Yield: $80 \%, \mathrm{Mp}: 153-155^{\circ} \mathrm{C},{ }^{1} \mathrm{H}$ NMR $\left(400 \mathrm{MHz}, \mathrm{CDCl}_{3}\right): \delta=9.38(\mathrm{~d}$, $1 \mathrm{H}) ; 8.15(\mathrm{~m}, 1 \mathrm{H}) ; 7.90(\mathrm{~s}, 2 \mathrm{H}) ; 7.62(\mathrm{~s}, 1 \mathrm{H}) ; 7.56(\mathrm{~m}, 2 \mathrm{H}) ; 7.45(\mathrm{~m}$, 1H); 7.30 (m, 2H); $7.21(\mathrm{~d}, 2 \mathrm{H}) ; 6.56(\mathrm{~m}, 2 \mathrm{H}) .{ }^{13} \mathrm{C}$ NMR $(100 \mathrm{MHz}$, $\left.\mathrm{CDCl}_{3}\right): \delta=158.9,156.3,148.0,142.3,135.5,131.3,128.2,122.2,120.2$, $119.4,118.5,111.3,108.1,105.3,102.3$. HRMS (ESI): $\mathrm{m} / \mathrm{z}[\mathrm{M}+\mathrm{H}]^{+}$calcd for $\mathrm{C}_{21} \mathrm{H}_{14} \mathrm{~N}_{2} \mathrm{O}_{2}$ : found: 327.6 .
2-[2,6-di(Thiophen-2-yl)pyridin-4-yl]-1H-indole(3ai): Colorless solid, Yield: $70 \%, \mathrm{Mp}: 169-171^{\circ} \mathrm{C},{ }^{1} \mathrm{H}$ NMR $\left(400 \mathrm{MHz}, \mathrm{CDCl}_{3}\right): \delta=9.12$ (d, $1 \mathrm{H}), 8.19(\mathrm{~s}, 1 \mathrm{H}), 8.07$ (s, 2H), $7.54(\mathrm{~d}, 1 \mathrm{H}), 7.49(\mathrm{~d}, 1 \mathrm{H}), 7.32(\mathrm{~d}$, 2H), $7.23(\mathrm{~m}, 1 \mathrm{H}), 7.15(\mathrm{~m}, 5 \mathrm{H}) .{ }^{13} \mathrm{CNMR}\left(100 \mathrm{MHz}, \mathrm{CDCl}_{3}\right): \delta=152.2$, 146.2, 137.4, 135.1, 129.9, 128.7, 128.3, 126.3, 122.9, 121.1, 119.2, 118.7, 111.6, 101.8. HRMS (ESI): $\mathrm{m} / \mathrm{z}[\mathrm{M}+\mathrm{H}]^{+}$calcd for $\mathrm{C}_{21} \mathrm{H}_{14} \mathrm{~N}_{2} \mathrm{~S}_{2}$ : found: 359. 7.

5-Bromo-3-(2,6-di(Phenylpyridin-4-yl))-1H-indole (3ba): White Solid, Yield: $72 \%, \mathrm{Mp}: 185-187^{\circ} \mathrm{C},{ }^{1} \mathrm{H}$ NMR $\left(400 \mathrm{MHz}, \mathrm{CDCl}_{3}\right): \delta=8.68$ (d, $1 \mathrm{H}), 8.23(\mathrm{~d}, 4 \mathrm{H}), 8.14(\mathrm{~s}, 1 \mathrm{H}), 7.90(\mathrm{~s}, 2 \mathrm{H}), 7.59(\mathrm{t}, 4 \mathrm{H}), 7.51(\mathrm{~d}$, $3 \mathrm{H}), 7.39(\mathrm{t}, 1 \mathrm{H}), 7.28(\mathrm{t}, 1 \mathrm{H}) .{ }^{13} \mathrm{C} \mathrm{NMR}\left(100 \mathrm{MHz}, \mathrm{CDCl}_{3}\right): \delta=157.6$, $144.2,139.8,135.4,129.0,128.7,127.2,127.0,125.9,124.4,122.2,117.1$, 115.9, 114.4, 113.2. HRMS (ESI): $\mathrm{m} / \mathrm{z}[\mathrm{M}+\mathrm{H}]^{+}$calcd for $\mathrm{C}_{25} \mathrm{H}_{17} \mathrm{~N}_{2} \mathrm{Br}_{2}$ : found: 426.9 .

3-(2,6-bis(4-Methoxyphenyl)pyridin-4-yl)-5-bromo- $1 \mathrm{H}$ indole(3bc): Colorless solid, Yield: $80 \% \mathrm{Mp}: 230-232^{\circ} \mathrm{C},{ }^{1} \mathrm{H}$ NMR $(400$ $\left.\mathrm{MHz}, \mathrm{CDCl}_{3}\right): \delta=8.64(\mathrm{~d}, 1 \mathrm{H}), 8.26(\mathrm{~d}, 4 \mathrm{H}) ; 7.92(\mathrm{~s}, 1 \mathrm{H}), 7.86(\mathrm{~s}, 2 \mathrm{H})$, $7.31(\mathrm{~d}, 2 \mathrm{H}), 7.29(\mathrm{~d}, 1 \mathrm{H}), 7.17(\mathrm{~d}, 4 \mathrm{H}), 3.7(\mathrm{~s}, 6 \mathrm{H}) .{ }^{13} \mathrm{C}$ NMR $(100 \mathrm{MHz}$, $\left.\mathrm{CDCl}_{3}\right): \delta=156.7,144.3,138.2,135.6,135.0,130.6,129.1,126.9,125.4$, 123.2, 119.6, 115.8, 115.1, 112.8, 111.5, 55.8. HRMS (ESI): $\mathrm{m} / \mathrm{z}[\mathrm{M}+\mathrm{H}]^{+}$ calcd for $\mathrm{C}_{27} \mathrm{H}_{21} \mathrm{~N}_{2} \mathrm{O}_{2}$ : found: 486.8 .

3-(2,6-bis (4-Chlorophenyl)pyridin-4-yl)-5-bromo- $1 \mathrm{H}$ indole(3bd): White Solid, Yield: $81 \%, \mathrm{mp} 120-122^{\circ} \mathrm{C},{ }^{1} \mathrm{H}$ NMR $(400$ $\left.\mathrm{MHz}, \mathrm{CDCl}_{3}\right): \delta=8.64(\mathrm{~d}, 1 \mathrm{H}), 8.14(\mathrm{~d}, 4 \mathrm{H}), 8.11-8.09$ (s, $\left.1 \mathrm{H}\right), 7.85$ (s, 2H), $7.57(\mathrm{~d}, 1 \mathrm{H}), 7.42(\mathrm{~d}, 4 \mathrm{H}), 7.40(\mathrm{~d}, 2 \mathrm{H}) .{ }^{13} \mathrm{C} \mathrm{NMR}(100 \mathrm{MHz}$, $\left.\mathrm{CDCl}_{3}\right): \delta=156.4,144.5,137.9,135.4,135.2,128.9,128.3,126.9,126.0$, 124.4, 122.1, 116.9, 115.6, 114.5, 113.2. HRMS (ESI): $\mathrm{m} / \mathrm{z}[\mathrm{M}+\mathrm{H}]^{+} \mathrm{calcd}$ for $\mathrm{C}_{25} \mathrm{H}_{15} \mathrm{~N}_{2} \mathrm{Cl}_{2} \mathrm{Br}$ : found: 494.8 .

3-(2,6-bis(4-Bromophenyl)pyridin-4-yl)-5-bromo-1H-indole (3be): White Solid, Yield: $83 \%$, Mp: $225-227^{\circ} \mathrm{C},{ }^{1} \mathrm{H}$ NMR $(400 \mathrm{MHz}$, $\left.\mathrm{CDCl}_{3}\right): \delta=8.57(\mathrm{~d}, 1 \mathrm{H}), 8.10(\mathrm{~d}, 4 \mathrm{H}), 8.05(\mathrm{~s}, 1 \mathrm{H}), 7.87(\mathrm{~s}, 2 \mathrm{H}), 7.67$ (d, $4 \mathrm{H}), 7.59$ (d, 1H), $7.43(\mathrm{~d}, 1 \mathrm{H}), 7.41(\mathrm{~d}, 1 \mathrm{H}) .{ }^{13} \mathrm{C}$ NMR $(100 \mathrm{MHz}$, $\left.\mathrm{CDCl}_{3}\right): \delta=156.5,144.5,138.3,135.4,131.9,128.6,126.9,126.1,124.4$, 123.6, 122.1, 117.0, 115.7, 114.5, 113.2. HRMS (ESI): $\mathrm{m} / \mathrm{z}[\mathrm{M}+\mathrm{H}]^{+} \mathrm{calcd}$ for $\mathrm{C}_{25} \mathrm{H}_{15} \mathrm{~N}_{2} \mathrm{Br}_{3}$ : found: 584.5 .

3-(2,6-bis(4-Fluorophenyl)pyridin-4-yl)-5-bromo-1H-indole (3bf): White Solid, Yield: $73 \%$, Mp: $219-221^{\circ} \mathrm{C},{ }^{1} \mathrm{H}$ NMR $(400 \mathrm{MHz}$, $\mathrm{CDCl}_{3}$ ): $\delta=8.59(\mathrm{~d}, 1 \mathrm{H}), 8.21(\mathrm{~d}, 4 \mathrm{H}), 8.18(\mathrm{~s}, 1 \mathrm{H}), 7.85(\mathrm{~s}, 2 \mathrm{H}), 7.61(\mathrm{~d}$, $1 \mathrm{H}), 7.43(\mathrm{~d}, 2 \mathrm{H}), 7.25$ (d, 4H). ${ }^{13} \mathrm{C} \mathrm{NMR}\left(100 \mathrm{MHz}, \mathrm{CDCl}_{3}\right): \delta=164.8$, $156.5,144.4,135.7,135.4,128.9,126.9,126.0,124.3,122.1,116.6,115.9$, 115.7, 114.5, 113.2. HRMS (ESI): $\mathrm{m} / \mathrm{z}[\mathrm{M}+\mathrm{H}]^{+}$calcd for $\mathrm{C}_{25} \mathrm{H}_{15} \mathrm{~N}_{2} \mathrm{BrF}_{2}$ : found: 462.8 .

\section{General experimental procedure for biological activity}

5-Lipoxygenase enzyme inhibitory activity: The indolylpyridines were screened for their 5-LOX inhibitory potential using colorimetric method. The assay mixture contained $50 \mathrm{mM}$ phosphate buffer, $\mathrm{pH}$ 6.3, 5-lipoxygenase, various concentrations of test substances in dimethylsulfoxide, and linoleic acid $(80 \mathrm{mM})$ in a total volume of 0.5 $\mathrm{mL}$, after $5 \mathrm{~min}$ incubation of the above reaction mixture, $0.5 \mathrm{~mL}$ ferric xylenol orange reagent (in perchloric acid) was added and absorbance was measured after two minutes at $585 \mathrm{~nm}$ on a spectrophotometer. Controls were run along with test in a similar manner, except using vehicle instead of test substance solution. Percent inhibition was calculated by comparing the absorbance values of the test solution with that of control. All the tests were run in triplicate and averaged. 


\section{Result and Discussion}

\section{Chemistry}

1H-Indole-3-carboxaldehyde and

5-bromo-1H-indole-3carboxaldehyde were prepared from indole using phosphorus oxychloride in DMF. The general synthesis of 2,6-diaryl-4indolylpyridines (3aa-3bf) is illustrated in Scheme 1. The reaction of indole-3-carboxaldehyde (1a-b) with substituted acetophenones (2a-i) in the presence of ammonium acetate in acetic acid at reflux conditions furnished 2,6-diaryl-4-indolylpyridines (3aa-3bf) in 63-84\% yield. Based on this protocol we have prepared 14 derivatives of and all the compounds were purified by column chromatography on silica gel. The chemical structures of the target compounds were confirmed by ${ }^{1} \mathrm{H}$ NMR, ${ }^{13} \mathrm{C}$ NMR, and MS spectra (Table 1).

\section{Biological activity}

5-Lipoxnase enzyme inhibitory activity: All the synthesized 2,6-diaryl-4-indolylpyridines (3aa-3bf) were screened for their 5-lipoxygenase enzyme inhibitory activity using colorimetric method [35] at different concentrations and found to have significant 5-LOX inhibitory activity with $\mathrm{IC}_{50}$ range 14.40 to $32.78 \mu \mathrm{g} / \mathrm{ml}$ (Table 2). Among all the compounds chloro substituted 2,6-diaryl-4indolylpyridine (3ad) $\left(\mathrm{IC}_{50} ; 14.40 \mu \mathrm{g} / \mathrm{ml}\right)$ and unsubstituted 2,6-diaryl4-indolylpyridine ( $3 \mathrm{aa})\left(\mathrm{IC}_{50} ; 17.40 \mu \mathrm{g} / \mathrm{ml}\right.$ ) showed very good activity whereas the compounds $3 \mathrm{bd}, 3 \mathrm{ba}, 3 \mathrm{bc}, 3 \mathrm{be}, 3 \mathrm{ae}, 3 \mathrm{bf}, 3 \mathrm{ab}$ and $3 \mathrm{ac}$ showed moderate activity. The compounds $3 a g$, 3 ai and 3 ah showed the least activity. In conclusion, we have synthesized a series of 2,6-diaryl substituted -4-indolylpyridine derivatives using commercially available<smiles>[R]c1ccc2[nH]cc(C=O)c2c1</smiles>

$\mathrm{R}=\mathrm{H}, \mathrm{Br}$<smiles>[R]c1ccc(C(C)=O)cc1</smiles>

Scheme 1: Synthesis of substituted 2, 6-diaryl-4-indolylpyridines.

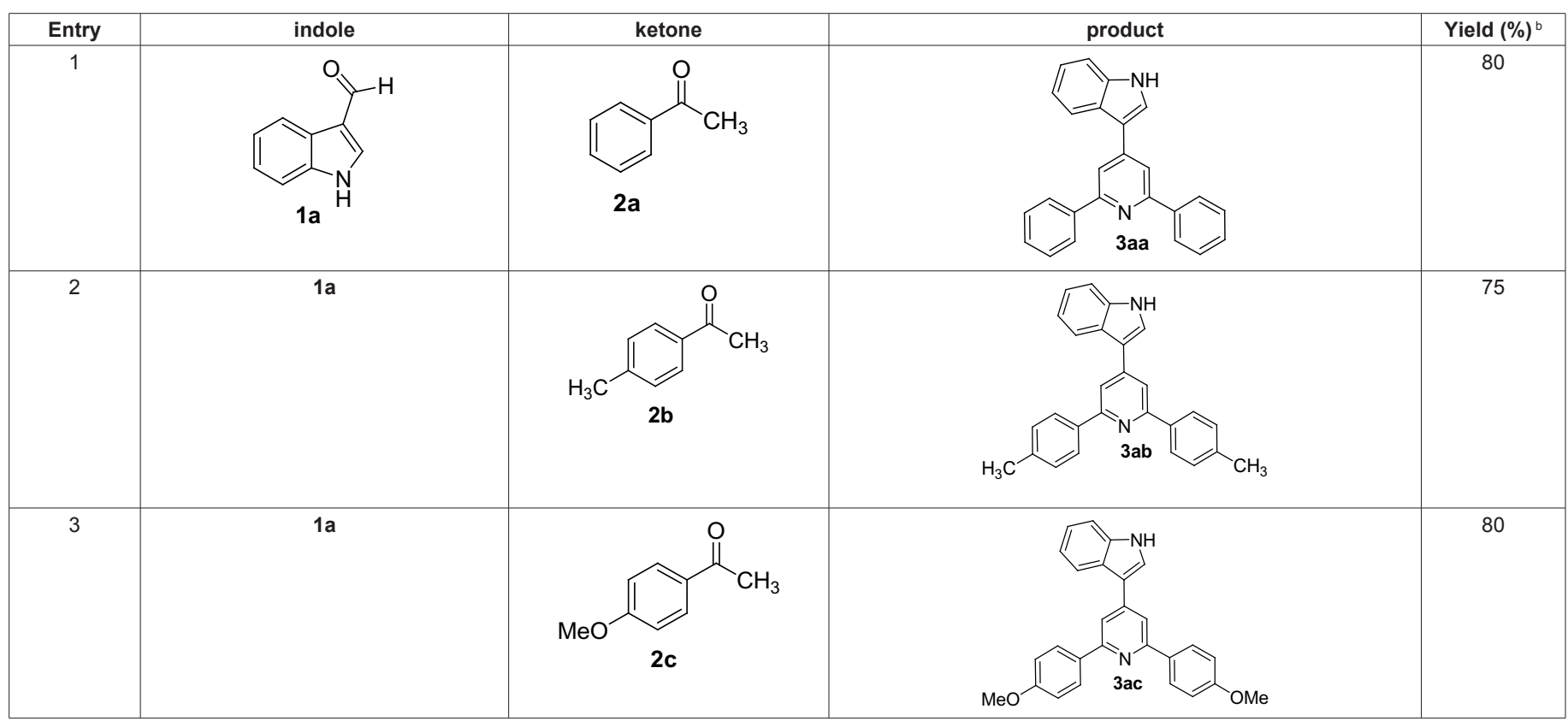




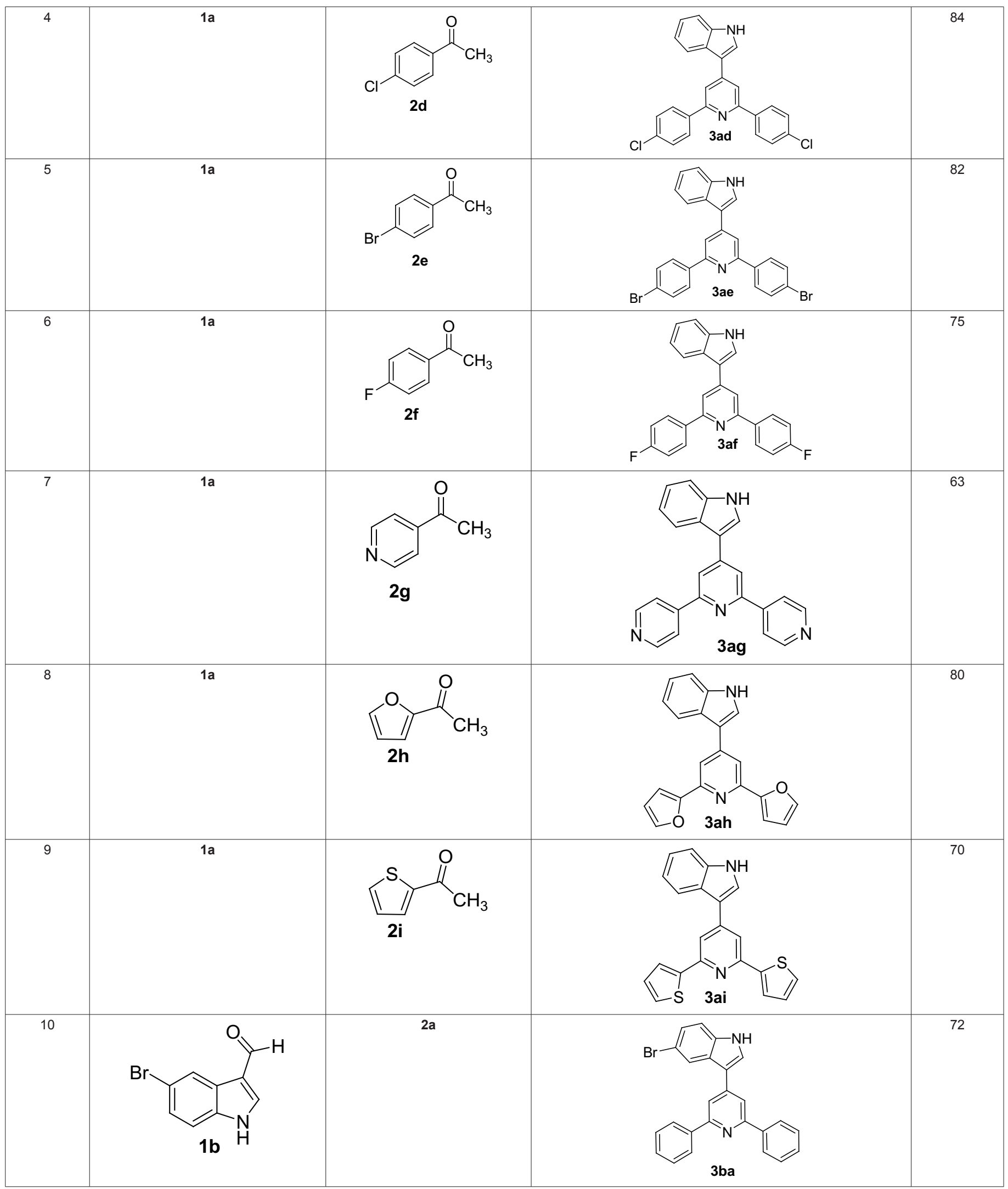


Citation: Tekluu B, Kadiri SK, Vidavalur S (2017) Synthesis of 2,6-Diaryl-4-Indolylpyridines as Novel 5-LOX Inhibitors. Med Chem (Los Angeles) 7: 894-899. doi: 10.4172/2161-0444.1000449

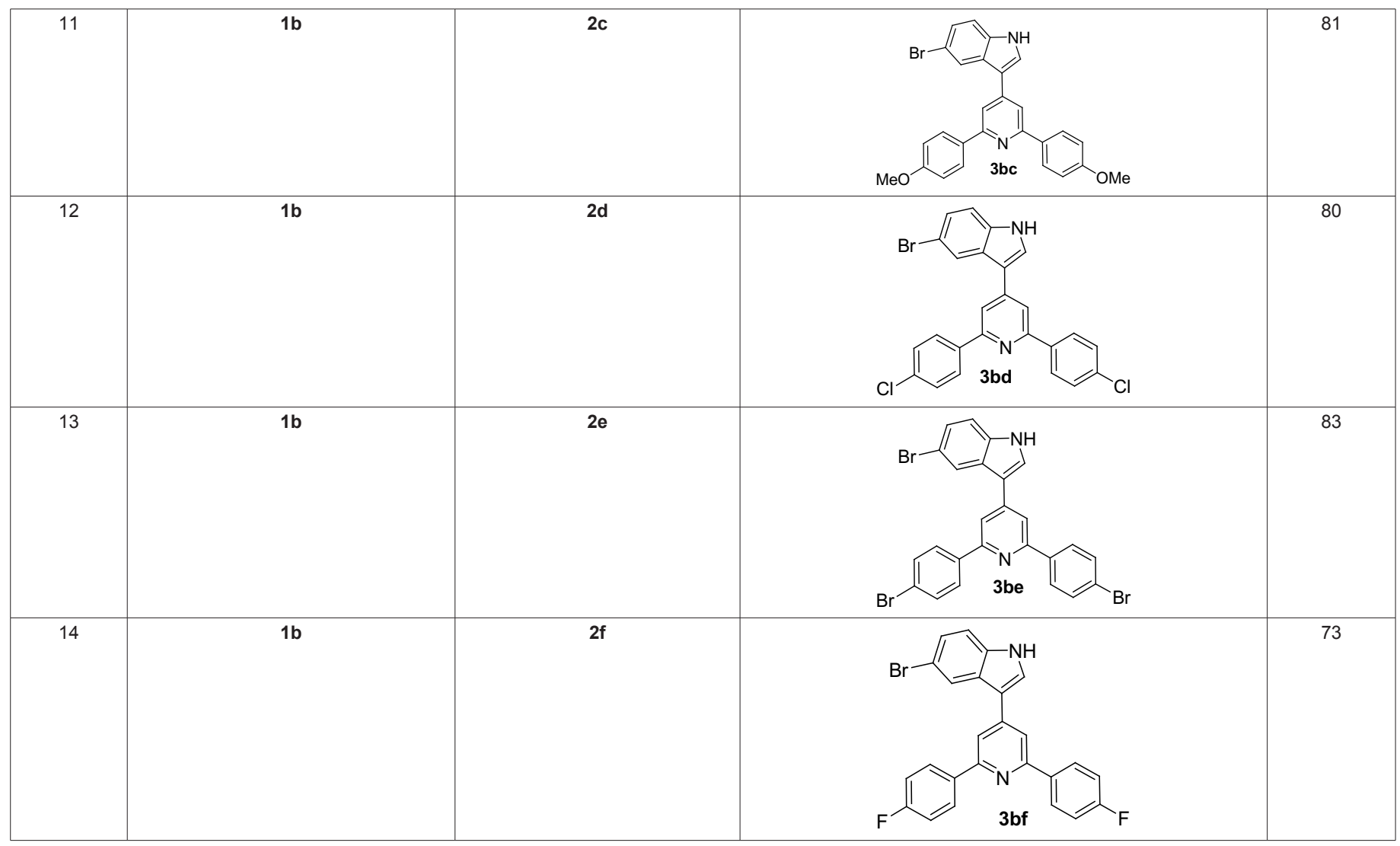

${ }^{\mathrm{b}}$ Isolated yields

Table 1: Synthesis of 2,6-diaryl-4-indolylpyridines ${ }^{b}$.

\begin{tabular}{|c|c|c|c|}
\hline Entry & Compound & Test items & IC $_{50} \boldsymbol{\mu M}$ \\
\hline 1 & $3 \mathrm{aa}$ & LNO-17-0001 & 17.40 \\
\hline 2 & $3 \mathrm{ab}$ & LNO-17-0002 & 32.95 \\
\hline 3 & $3 \mathrm{ac}$ & LNO-17-0003 & 33.14 \\
\hline 4 & $3 \mathrm{ad}$ & LNO-17-0004 & 14.40 \\
\hline 5 & $3 \mathrm{ae}$ & LNO-17-0005 & 29.94 \\
\hline 6 & $3 \mathrm{af}$ & LNO-17-0006 & $>100$ \\
\hline 7 & $3 \mathrm{ag}$ & LNO-17-0007 & 34.56 \\
\hline 8 & $3 \mathrm{ah}$ & LNO-17-0008 & 42.62 \\
\hline 9 & $3 \mathrm{ai}$ & LNO-17-0009 & 38.65 \\
\hline 10 & $3 \mathrm{ba}$ & LNO-17-0010 & 24.83 \\
\hline 11 & $3 \mathrm{bc}$ & LNO-17-0011 & 25.21 \\
\hline 12 & 3bd & LNO-17-0012 & 21.05 \\
\hline 13 & 3be & LNO-17-0013 & 25.78 \\
\hline 14 & 3bf & LNO-17-0014 & 32.78 \\
\hline Standard * & & & 36.49 \\
\hline
\end{tabular}

*Nordihydroguaiaretic acid

Table 2: $I C_{50}$ values obtained from in vitro 5-lipoxygenase inhibition assay for the compounds (3aa-3bf)

starting materials. 5-Lipoxygenase (5-LOX) enzyme inhibitory activities were performed for all the synthesized compounds. Among the tested compounds $3 \mathrm{ad}$ and $3 \mathrm{aa}$ showed good 5-lipoxygenase enzyme inhibitory activity.

\section{Acknowledgement}

The authors thank CSIR, New Delhi for financial assistance (through a project 02(0197)/14/EMR-II) and the Ministry of Education, Ethiopia, for financial support to B. T. G.

\section{References}

1. Gribble GW (2000) Recent developments in indole ring synthesis-methodology and applications. J Chem Soc Perkin Transactions 1: 1045-1075

2. Seefeld MA, Miller WH, Newlander KA, Burgess WJ, DeWolf WE, et al. (2003) Indole naphthyridinones as inhibitors of bacterial enoyl-ACP reductases Fabl and FabK. J Med Chem 46: 1627-1635.

3. Bhambi D, Salvi VK, Jat JL, Ojha S, Talesara GL (2007) Synthesis and antimicrobial activity of some new indole containing isoxazolines and phthalimidoxy derivatives of thiazolidinone and thiohydantoin. J Sulfur Chem 28: 155-163

4. Olomola TO, Bada DA, Obafemi CA (2009) Synthesis and antibacterial activity of two spiro [indole] thiadiazole derivatives. Toxicol Environ Chem 91: 941-946.

5. Joshi KC, Pathak VN, Arya P, Chand P (1979) Syntheses of some new fluorine containing indole derivatives and their antibacterial activity. Agric Biol Chem 43: 171-173.

6. Tiwari RK, Singh D, Singh J, Yadav V, Pathak AK, et al. (2006) Synthesis and antibacterial activity of substituted 1, 2, 3, 4-tetrahydropyrazino [1, 2-a] indoles. Bioorg Med Chem Lett 16: 413-416.

7. Chavan RS, More HN, Bhosale AV (2011) Synthesis, characterization and evaluation of analgesic and anti-inflammatory activities of some novel indoles. Trop J Pharmaceutica Res 10: 463-473.

8. Rani P, Srivastava VK, Kumar A (2004) Synthesis and anti-inflammatory activity of heterocyclic indole derivatives. Eur J Med Chem 39: 449-452.

9. Radwan MA, Ragab EA, Sabry NM, El-Shenawy SM (2007) Synthesis and biological evaluation of new 3-substituted indole derivatives as potential antiinflammatory and analgesic agents. Bioorg Med Chem 15: 3832-3841.

10. Chandra T, Garg N, Kumar A (2010) Synthesis and anti-inflammatory activity of indole derivatives. Int J Chem Tech Res 2: 762-773.

11. Tohid SFM, Ziedan NI, Stefanelli F, Fogli S, Westwell AD (2012) Synthesis and 
Citation: Tekluu B, Kadiri SK, Vidavalur S (2017) Synthesis of 2,6-Diaryl-4-Indolylpyridines as Novel 5-LOX Inhibitors. Med Chem (Los Angeles) 7: 894-899. doi: 10.4172/2161-0444.1000449

evaluation of indole-containing 3, 5-diarylisoxazoles as potential pro-apoptotic antitumors agents. Eur J Med Chem 56: 263-270.

12. Asma AA, Hanadi YM, Hanan FA, Naeema HY, Abdellatif MS (2014) In Vitro Antitumor and Antioxidant Activity of Meridianin Derivatives Synthesized from Indolylenaminonitriles under Microwave Irradiation. Am J Chem Appl 1: 6-11

13. Baraldi PG, Romagnoli R, Beria I, Cozzi P, Geroni C, et al. (2000) Synthesis and antitumor activity of new benzo heterocyclic derivatives of distamycin A. J Med Chem 43: 2675-2684.

14. Hung NC, Lhoste JM, Lavelle F, Bissery MC, Bisagni E (1990) Synthesis and antitumor activity of 1-[((dialkylamino) alkyl] amino]-4-methyl-5H-pyrido [4, 3-b] benzo [e]-and-benzo [g]) indoles. A new class of antineoplastic agents. J Med Chem 33: 1519-1528.

15. Panathur N, Dalimba U, Koushik PV, Alvala M, Yogeeswari P, et al. (2013) Identification and characterization of novel indole based small molecules as anticancer agents through SIRT1 inhibition. Eur J Med Chem 69: 125-138.

16. Sharma V, Kalia R, Raj T, Gupta VK, Suri N, et al. (2012) Synthesis and cytotoxic evaluation of substituted 3-(3'-indolyl-/3'-pyridyl)-isoxazolidines and bis-indoles. Acta Pharmaceutica Sinica B 2: 32-41.

17. Routier S, Mérour JY, Dias N, Lansiaux A, Bailly C, et al. (2006) Synthesis and biological evaluation of novel phenylcarbazoles as potential anticancer agents. J Med Chem 49: 789-799.

18. Rao VK, Chhikara BS, Shirazi AN, Tiwari R, Parang K, et al. (2011) 3-Substitued indoles: one-pot synthesis and evaluation of anticancer and Src kinase inhibitory activities. Bioorg Med Chem Lett 21: 3511-3514.

19. Safdy ME, Kurchacova E, Schut RN, Vidrio H, Hong E (1982) Tryptophan analogs. Synthesis and antihypertensive activity of positional isomers. J Med Chem 25: 723-730.

20. Nagai $Y$, Irie A, Masuda $Y$, Oka M, Uno H (1979) Synthesis of 2, 3, 4, 4a, 5 , $9 \mathrm{~b}$-hexahydro-1H-pyrido [4, 3-b] indole derivatives and their central nervous system activities. J Med Chem 22: 677-683.

21. Williams JD, Chen JJ, Drach JC, Townsend LB (2004) Synthesis and antivira activity of 3-formyl-and 3-cyano-2, 5, 6-trichloroindole nucleoside derivatives. J Med Chem 47: 5766-5772.

22. Zhao C, Zhao Y, Chai H, Gong $P$ (2006) Synthesis and in vitro anti-hepatitis $B$ virus activities of some ethyl 5-hydroxy-1H-indole-3-carboxylates. Bioorg Med Chem 14: 2552-2558.

23. deOliveira MR, Torres JC, Garden SJ, dos Santos CVB, Alves TR, et al. (2002)
Synthesis and antiviral evaluation of isatin ribonucleosides. Nucleosides, Nucleotides and Nucleic Acids 21: 825-835.

24. Bal TR, Anand B, Yogeeswari P, Sriram D (2005) Synthesis and evaluation of anti-HIV activity of isatin $\beta$-thiosemicarbazone derivatives. Bioorg Med Chem Lett 15: 4451-4455.

25. Sechi M, Derudas M, Dallocchio R, Dessi A, Bacchi A, et al. (2004) Design and synthesis of novel indole $\beta$-diketo acid derivatives as HIV-1 integrase inhibitors. J Med Chem 47: 5298-5310.

26. Perrier V, Wallace AC, Kaneko K, Safar J, Prusiner SB, et al. (2000) Mimicking dominant negative inhibition of prion replication through structure-based drug design. Proceedings of the National Academy of Sciences 97: 6073-6078.

27. Chen H, Zhang W, Tam R, Raney AK (2005) Thiazolidinones, oxazolidinones and pyrrolidinones for HBV. PCT Int Appl WO 2005058315 A1 20050630.

28. Reck F, Zhou F, Eyermann CJ, Kern G, Carcanague D, et al. (2007) Novel substituted (pyridin-3-yl) phenyloxazolidinones: antibacterial agents with reduced activity against monoamine oxidase $\mathrm{A}$ and increased solubility. $\mathrm{J}$ Med Chem 50: 4868-4881.

29. Zhao LX, Moon YS, Basnet A, Kim EK, Jahng Y, et al. (2004) Synthesis, topoisomerase I inhibition and structure-activity relationship study of 2, 4, 6-trisubstituted pyridine derivatives. Bioorg Med Chem Lett 14: 1333-1337.

30. Cabrera DG, Douelle F, Younis Y, Feng TS, Le Manach C, et al. (2012) Structure-Activity Relationship Studies of Orally Active Antimalarial 3, 5-Substituted 2-Aminopyridines. J Med Chem 55: 11022-11030.

31. Xiong WN, Yang CG, Jiang B (2001) Synthesis of novel analogues of marine indole alkaloids: Mono (indolyl)-4-trifluoromethylpyridines and bis (indolyl)-4 trifluoromethylpyridines as potential anticancer agents. Bioorg Med Chem 9: 1773-1780.

32. Thirumurugan P, Mahalaxmi S, Perumal PT (2010) Synthesis and antiinflammatory activity of 3 -indolylpyridine derivatives through one-pot multi component reaction. J Chem Sci 122: 819-832.

33. Samuelsson B (1983) The leukotrienes: Mediators of immediate hypersensitivity reactions and inflammation. In Leukotrienes and Prostacyclin 15-41.

34. Ghosh J, Myers CE (1998) Inhibition of arachidonate 5-lipoxygenase triggers massive apoptosis in human prostate cancer cells. Proceedings of the National Academy of Sciences 95: 13182-13187.

35. Gay CA, Gebicki JM (2002) Perchloric acid enhances sensitivity and reproducibility of the ferric-xylenol orange peroxide assay. Anal Biochem 304 $42-46$. 